\title{
Ping Feng Qingfei mixture treats airway hyperresponsiveness: a network pharmacology and molecular docking study
}

\author{
Min Wang ${ }^{1 \#}$, Min Peng ${ }^{2 \#}$, Hongshuo Shi ${ }^{3}$, Chengda Dong ${ }^{3}$, Lujie Cui ${ }^{4}$, Hui Chang ${ }^{3}$, Zunqi Kan ${ }^{3}$, \\ Kun Zhen ${ }^{3}$, Guomin $\mathrm{Si}^{2}$, Hui $\mathbf{L i}^{5}$ \\ ${ }^{1}$ Experimental Center, Shandong University of Traditional Chinese Medicine, Jinan, China; ${ }^{2}$ Department of Traditional Chinese Medicine, \\ Shandong Provincial Hospital Affiliated to Shandong First Medical University, Jinan, China; ${ }^{3}$ College of Traditional Chinese Medicine, Shandong \\ University of Traditional Chinese Medicine, Jinan, China; ${ }^{4}$ First Clinical Medical College, Shandong University of Traditional Chinese Medicine, \\ Jinan, China; ${ }^{5}$ Department of Critical Care, Affiliated Hospital of Shandong University of Traditional Chinese Medicine, Jinan, China \\ Contributions: (I) Conception and design: M Wang, M Peng, H Li; (II) Administrative support: M Wang; (III) Provision of study materials or patients: \\ L Cui; (IV) Collection and assembly of data: H Chang; (V) Data analysis and interpretation: Z Kan, K Zhen; (VI) Manuscript writing: All authors; (VII) \\ Final approval of manuscript: All authors. \\ \#These authors contributed equally to this work. \\ Correspondence to: Hui Li. Department of Critical Care, Affiliated Hospital of Shandong University of Traditional Chinese Medicine, Jinan 250021, \\ China. Email: lihui_tcm@126.com.
}

Background As air pollution has increased in severity over recent years, fine particulate matter (PM) (<25 $\mu \mathrm{m}$; PM2.5) has led to a greater incidence of disease, including airway hyperresponsiveness (AHR). Ping Feng Qingfei Mixture (PFQF) is effective in treating AHR caused by PM2.5. As there is a lack of knowledge regarding the mechanisms of $\mathrm{PFQF}$ in the treatment of AHR, we conducted a network pharmacology study to clarify this issue.

Methods: We obtained the composition of PFQF from the traditional Chinese medicine (TCM) systems pharmacology database and its potential targets. The potential targets of AHR were obtained from the Online Mendelian Inheritance in Man and Gene Cards databases. Then psychophysiological interaction, KEGG pathway, and Gene Ontology biological process analyses were carried out for targeting PFQF in treating AHR. We further constructed a related network diagram and verified the experimental results in molecular docking.

Results: We identified a total of 4 core active compounds, and through KEGG analysis obtained multiple signaling pathways, including T helper17 (Th17) cell differentiation and interleukin-17 (IL-17) signaling pathway. Our molecular docking also verified that PFQF could effectively regulate the imbalance of Th17-T regulatory (Treg) cells.

Conclusions: PFQF can effectively treat the AHR caused by PM2.5 through Th17-Treg immune balance. The combination of molecular docking and network pharmacology provides a way to elucidate the complex mechanism of action of this Chinese herbal medicine.

Keywords: Network pharmacology; Ping Feng Qingfei mixture; PM2.5; airway hyperresponsiveness (AHR); molecular docking

Submitted Mar 16, 2021. Accepted for publication Apr 23, 2021.

doi: 10.21037/apm-21-802

View this article at: http://dx.doi.org/10.21037/apm-21-802 


\section{Introduction}

Airway hyperresponsiveness (AHR) is a response to excessive airway contraction caused by various inhalation stimuli, including chemical and physical stimuli (1). AHR is common in many respiratory diseases, such as asthma, allergic rhinitis, upper respiratory tract infection, acute and chronic bronchitis, etc. (2). Many scholars have explored the mechanism of AHR and found that there are many relevant factors, including gene regulation, airway inflammation, nerve factors, and airway smooth muscle and airway remodeling (3). Airway inflammation is the basis of AHR (4). In many developing countries, including China, people's living environment and health are increasingly affected by air pollution. Inhalable particulate matter $(\mathrm{PM})$ is an important factor leading to air pollution (5). Fine particles (PM2.5, particle size $<25 \mu \mathrm{m}$ ) are inhaled through the nasal cavity, collect in the lungs, and then enter the alveolar cavity, and may even enter circulation. This causes inflammation and immune responses in the lungs and throughout the body (6). Studies have shown that PM2.5 is closely related to the formation and aggravation of AHR and airway inflammation $(7,8)$. At present, there is no special diagnosis and treatment plan for haze, and anti-inflammatory, antiallergic, and drugs used to relieve spasm of the airway smooth muscles are the main treatment options for AHR. These can include bronchodilators and inhaled corticosteroids. Long-term use of these drugs can induce certain adverse reactions, leading to poor compliance. Glucocorticoid therapy has good anti-inflammatory and immunosuppressive effects, but its disadvantages are also obvious. Long-term use of glucocorticoids may cause complications such as hypertension, hyperglycemia, and liver failure (9). Given this, for the AHR caused by PM2.5, identifying the most effective Chinese herbal medicine may provide a more benign alternative treatment.

Chinese herbal medicines have been widely used in the treatment of a series of diseases for thousands of years. It has the advantages of low price and wide availability. Moreover, many herbal drugs are obtained from nature, so the side effects are minimal (10). Therefore, for the treatment of AHR, it is of great significance to identify the traditional Chinese medicine (TCM) compounds with most curative effect. Based on observations of the preparation of Ping Feng Gujin mixture in hospital during the period of severe acute respiratory syndrome (SARS), we prepared a mixture of effective prescriptions, named Ping feng Qingfei
(PFQF), to prevent lung injury. This medicinal mixture is composed of Hedysarum multijugum Maxim ("Huang Qi" in Chinese, or HQ), Phragmitis rhizoma ("Lu Gen" in Chinese, or LG), Pseudostellariae radix ("Tai Zi Shen" in Chinese, or TZS), Lilii bulbus ("Bai He" in Chinese, or $\mathrm{BH}$ ), Atractylodes macrocephala Koidz ("Bai Zhu" in Chinese, or BZ), Siraitia grosvenorii ("Luo Han Guo" in Chinese, or LHG), Perilla frutescens ("Zi Su" in Chinese, or ZS), and Saposhnikoviae radix ("Fang Feng" in Chinese, or FF), and has been successfully prepared in the hospital. Owing to its complex composition and different targets, the mechanism of PFQF is difficult fully elucidate. Here, for the first time, we combine molecular docking verification and methodology of network pharmacology prediction to study the therapeutic effects of PFQF.

Network pharmacology has gained a wide range of application value in drug target recognition, active ingredient study, action mechanism research, preclinical research of efficacy, safety evaluation, and some other areas (11). The purpose of our work was to make out the natural compounds of PFQF using network pharmacology and to explore key targets of PFQF in the treatment of AHR, so as to comprehend its molecular docking verification and potential mechanism of action. Our study plan is illustrated in Figure 1. We hope the experimental results of this study can promote awareness of $\mathrm{PFQF}$ and provide an alternative treatment for PM2.5-induced respiratory diseases.

\section{Methods}

\section{Screening of the active ingredients of $P F Q F$}

The compounds present in PFQF were selected from the TCMSP database. In order to identify the active components of $\mathrm{PFQF}$, the ingredients conforming to the requirements of both oral bioavailability $(\mathrm{OB}) \geq 30 \%$ and drug-likeliness (DL) $\geq 0.18$, based on the published literature and the information extracted from the TCMSP database, were identified for further analysis (12).

\section{Acquiring the target protein of the selected compounds}

All the active ingredients were input into the TCMSP database to obtain the known targets, while the 2D structure of the compounds obtained from PubChem were imported into the Swiss Target Prediction database. A threshold (probability, $\mathrm{P}>0.6$ ) was set to obtain more credible targets for each compound (13). 


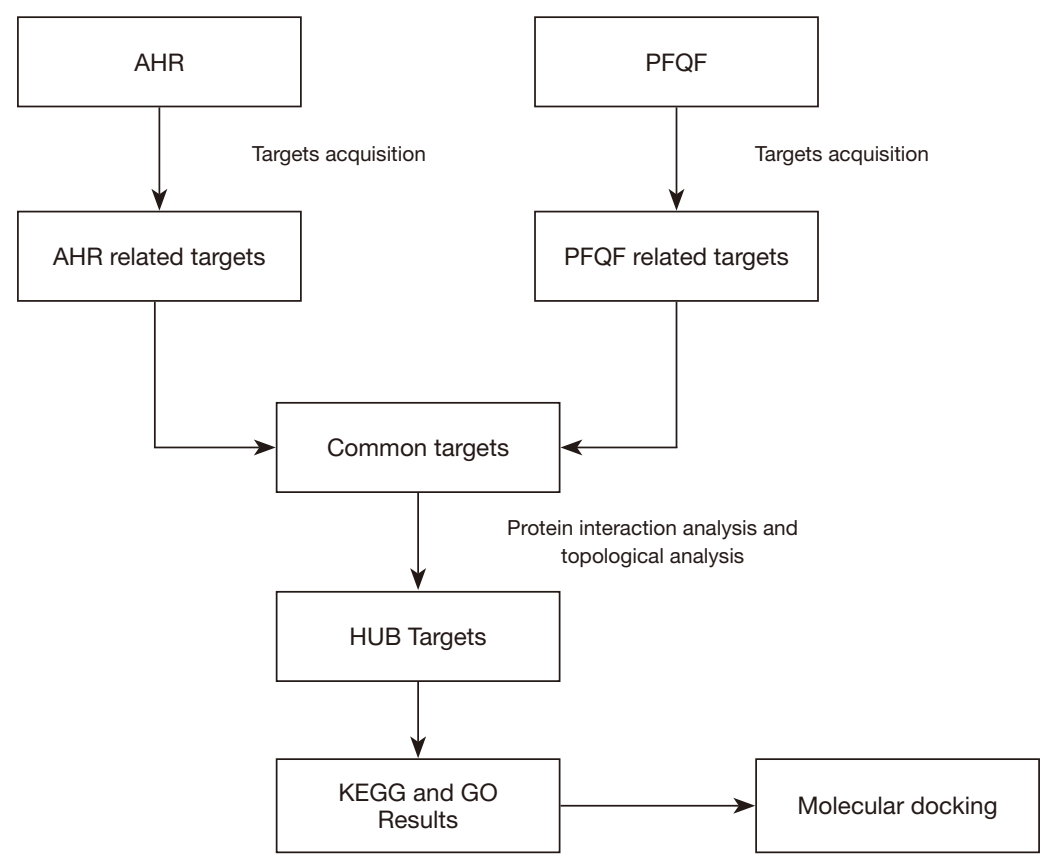

Figure 1 Study framework based on an integration strategy of network pharmacology.

\section{Search targets of AHR}

We selected the therapeutic target data from the following resources: Online Mendelian Inheritance in Man (OMIM), and Gene Cards human gene database. Furthermore, the official names of the genes (PFQF and AHR) were acquired from UniProt, a protein sequence database, with "Homo sapiens" being the selected species. The names of the targets were then modified into gene and UniProt ID (14). Finally, we mapped the target genes of PFQF active constituents and AHR-related target genes, and screened the coincident target genes as the related targets of PFQF treatment for AHR.

\section{Protein-protein interaction (PPI) network of target protein interaction}

Using the STRING database, we constructed a detectable target of PFQF anti-AHR protein interaction. STRING is a database of known and predicted PPIs which can reflect the interaction between proteins in vivo. The target protein of PFQF against AHR was amplified using the STRING database, and we input them into Cytoscape software. We used the "Analysis Network" tool in Cytoscape to construct the PPI network and obtain the relevant parameters. Based on the 4 parameters of degree, closeness centrality, betweenness centrality, and stress, we performed a topology analysis on the PPI network to acquire the hub nodes.

\section{Enrichment analysis of Gene Ontology and Kyoto Encyclopedia of Genes and Genomes pathway}

After topology analysis, the hub target was used to analyze the enrichment of KEGG pathway through the Cytoscape plugins, ClueGo and CluePedia. We further analyzed the enrichment of hub genes in Gene Ontology (GO) biological process. We input the related target genes of PFQF for treating AHR into DAVID (Database for Annotation, Visualization and Integrated Discovery) v. 6.8.

\section{Molecular docking}

Using LeDock software (LePhar), we conducted a simulation of the receptor ligand docking of the core target in KEGG pathway with the main active compounds in the first 5 positions of degree. A binding energy less than 0 indicates the stable bound between ligand and receptor molecules. The larger the absolute value, the stronger the stability. If the binding energy was more than 0 , this indicated that the conformation of the ligand and receptor molecules could not be bound stably, and a higher absolute value represent more instability of the conformation (15). 


\section{Statistical analysis}

The pathway information of the selected targets was retrieved from the KEGG pathway enrichment analysis. The topological data were analyzed by Cytoscape version 3.6.0. for Windows. Pathway enrichment analysis was performed using DAVID v. 6.8 .

The study was conducted in accordance with the Declaration of Helsinki (as revised in 2013).

\section{Results}

\section{Screening of compounds and acquisition of targets}

73 active compounds were screened according to chemical similarity and acquired 225 (after deleting the duplicates) related targets. As shown in Figure 2A, we constructed a herbal-compound-target network diagram comprising 306 nodes and 1,234 edges. We used the "Analysis Network" tool in Cytoscape to acquire the degree parameter of the network. According to this network diagram, we found that the top 3 compounds of degree parameter were quercetin (MOL000098), kaempferol (MOL000422), $\beta$-sitosterol (MOL000358), and stigmasterol (MOL000449) (Table 1).

\section{PPI network analysis}

The AHR target data in OMIM and GeneCards databases were integrated with a multisource database integration method. A total of 809 AHR targets were obtained after removal of duplicate values. The intersection of $\mathrm{PFQF}$ and AHR-related targets were collected, and 83 common targets were obtained as the related targets of PFQF in AHR. We input these 83 target genes into the STRING database to construct a PPI network diagram of 585 nodes. The obtained targets were divided into 2 parts, including 585 targets (83 public targets and 402 other human protein targets), which represented the $\mathrm{PFQF}$ in vivo response to AHR. In total, there were 585 nodes and 16,771 edges (Figure $2 B$ ). After this, the topological characteristics of the PPI network were analyzed. Based on the 4 parameters of DC (degree centrality), BC (betweenness centrality), CC (closeness centrality), and stress, the index above the median value was selected as the key index [27] (Figure 3) and the core nodes of PFQF acting on AHR were obtained. The threshold values of the first screening were degree $\geq 44$, closeness centrality $\geq 0.475958$, betweenness centrality $\geq 0.000701$, and stress $\geq 8,960$. Topological analysis and data screening were then repeated, and 85 hub targets were obtained. The criteria for screening were degree $\geq 64.5$, closeness centrality $\geq 0.585431$, betweenness centrality $\geq 0.001343$, and stress $\geq 1,458$. We acquired 95 hub nodes and constructed the related network diagram. The network diagram (Figure 4A) comprised 95 nodes and 2,866 edges (Table 2).

\section{Enrichment analysis of KEGG pathway and GO biological process (Figure 4B)}

\section{KEGG pathway analysis}

The enrichment analysis of the KEGG pathway was performed, and 95 HUB targets were derived through ClueGO and CluePedia. A total of 102 KEGG pathways were obtained by enrichment analysis $(\mathrm{P}<0.05)$. The results show that the metabolic pathways were mainly involved in T helper17 (Th17), T helper1 (Th1), and T helper2 (Th2) cell differentiation, and interleukin-17 (IL-17) signaling pathway. From this, we speculate that PFQF could play a role by regulating the Th17-T regulatory (Treg) cells imbalance.

\section{GO analysis}

After enrichment analysis of 95 hub targets using DAVID v. 6.8, we identified $106 \mathrm{GO}$ biological processes according to $\mathrm{P}$ value $<0.01$. We obtained the first $20 \mathrm{GO}$ biological processes for further analysis, and found that PFQF treats AHR by regulating cell proliferation, apoptosis, inflammation, and angiogenesis.

\section{Molecular docking}

Core compounds in the first 4 positions of LeDock were used for molecular docking with Foxp3 and RoR $\gamma$ t. The results showed that the binding energies of all molecules to proteins were less than 0 , indicating that ligands could spontaneously bind to receptors (Figure 5).

\section{Discussion}

Epidemiological research has shown that the increase of PM2.5 in the air is closely related to the aggravation of AHR symptoms (16). Further studies have found that the current treatment for AHR, even long-term application of medication, cannot completely prevent airway remodeling. Chinese herbal medicine has shown unique advantages in the treatment of haze injury, and could be used as a supplementary treatment of AHR. In the process of 


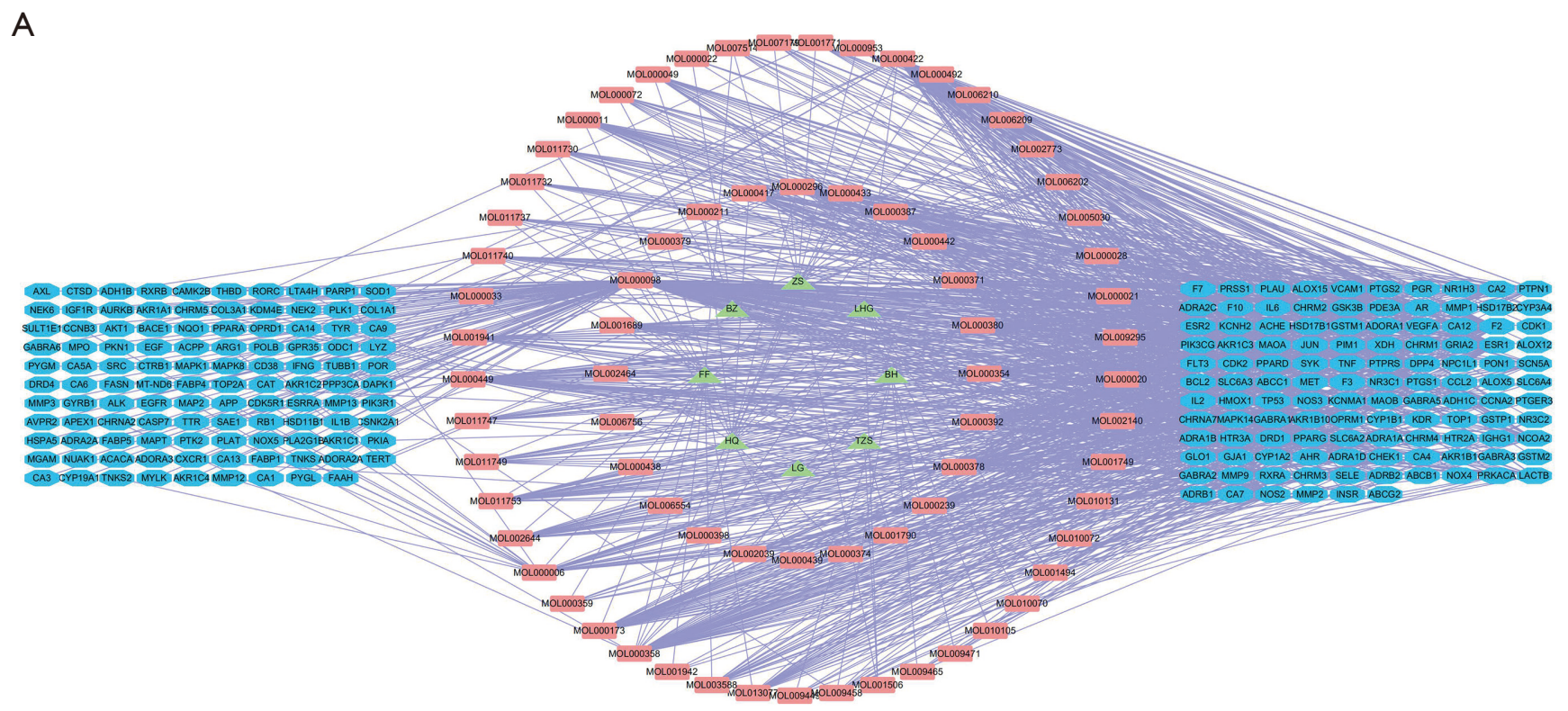

B

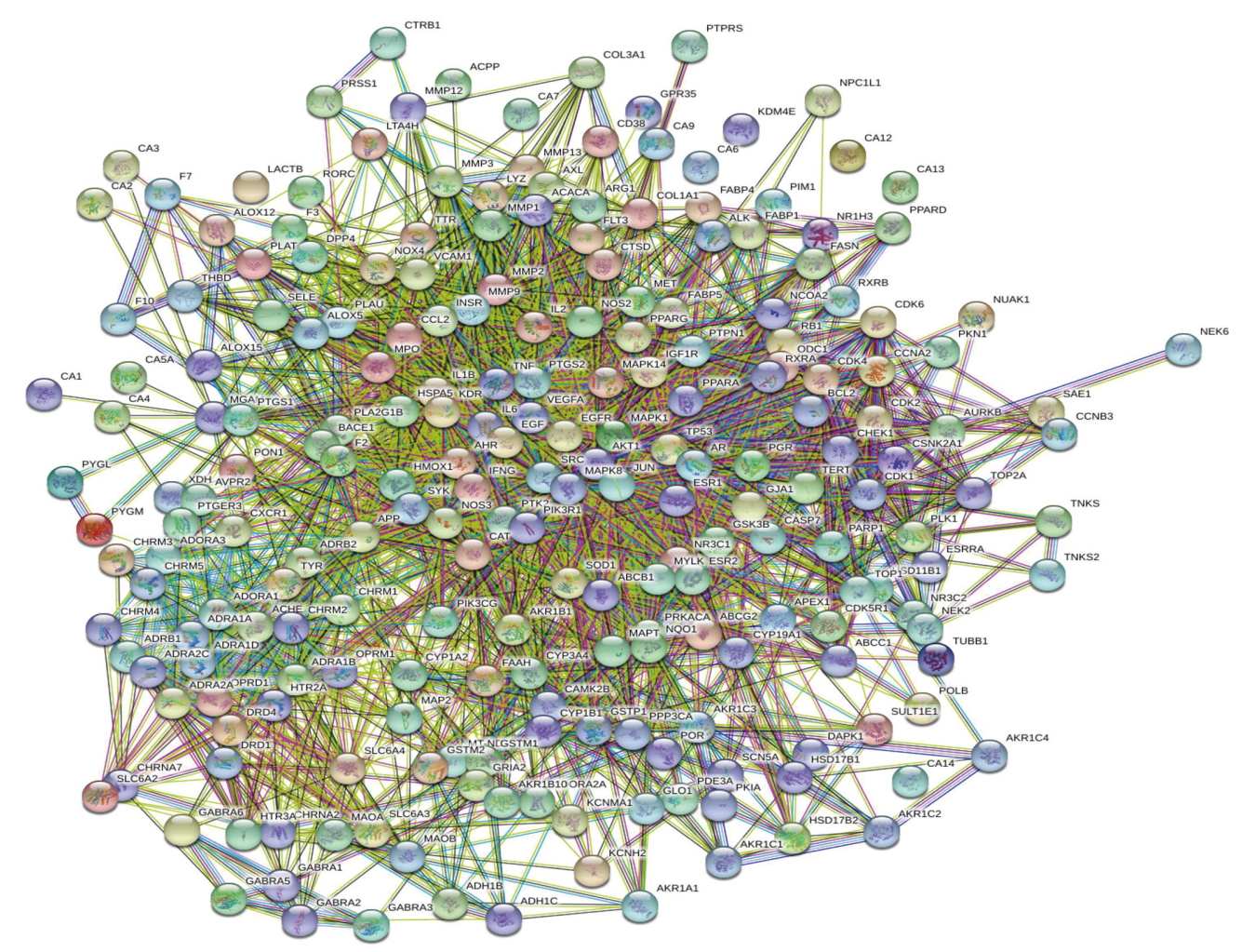

Figure 2 Herbal-compound-target network and PPI network. (A) The herbal-compound-target network of PFQF in AHR treatment. Green represents Chinese herbal medicine, red and orange represent compounds, and blue represents targets. The triangle represents Chinese herbal medicine, the rectangle represents compounds, the hexagon represents targets. (B) PPI network diagram of targets of traditional Chinese medicine. PFQF, Ping Feng Qingfei Mixture; AHR, airway hyperresponsiveness; PPI, protein-protein interaction. 
Table 1 Compound information sheet

\begin{tabular}{|c|c|c|c|c|c|}
\hline Molecule ID & Molecule name & Structure & OB & $\mathrm{DL}$ & Herb \\
\hline Kaempferol & MOL000422 & & $41.88 \%$ & 0.24 & HQ, LHG \\
\hline Stigmasterol & MOL000449 & & $43.83 \%$ & 0.76 & LG, BH \\
\hline
\end{tabular}

OB, oral bioavailability; DL, drug-likeliness; HQ, Huang Qi; LHG, Luo Han Guo; TZS, Tai Zi Shen; BH, Bai He; ZS, Zi Su; FF, Fang Feng; LG, Lu Gen.

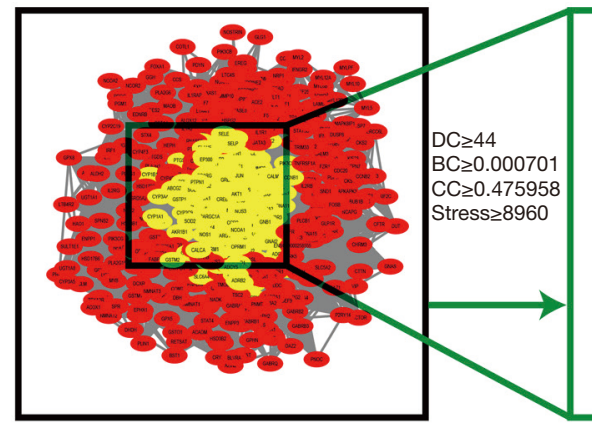

585 nodes and 16771 edges

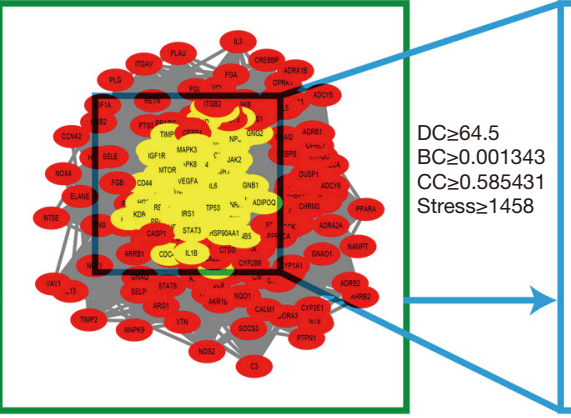

222 nodes and 7765 edges

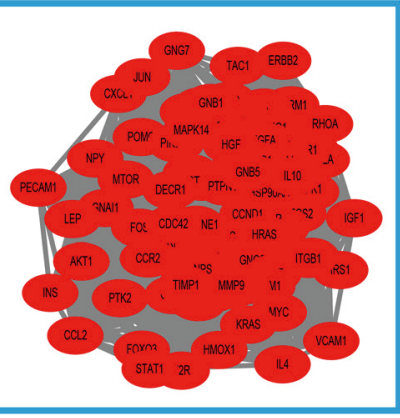

95 nodes and 2866 edges

Figure 3 Network topology filter map. DC, degree centrality; BC, betweenness centrality; CC, closeness centrality. 
A

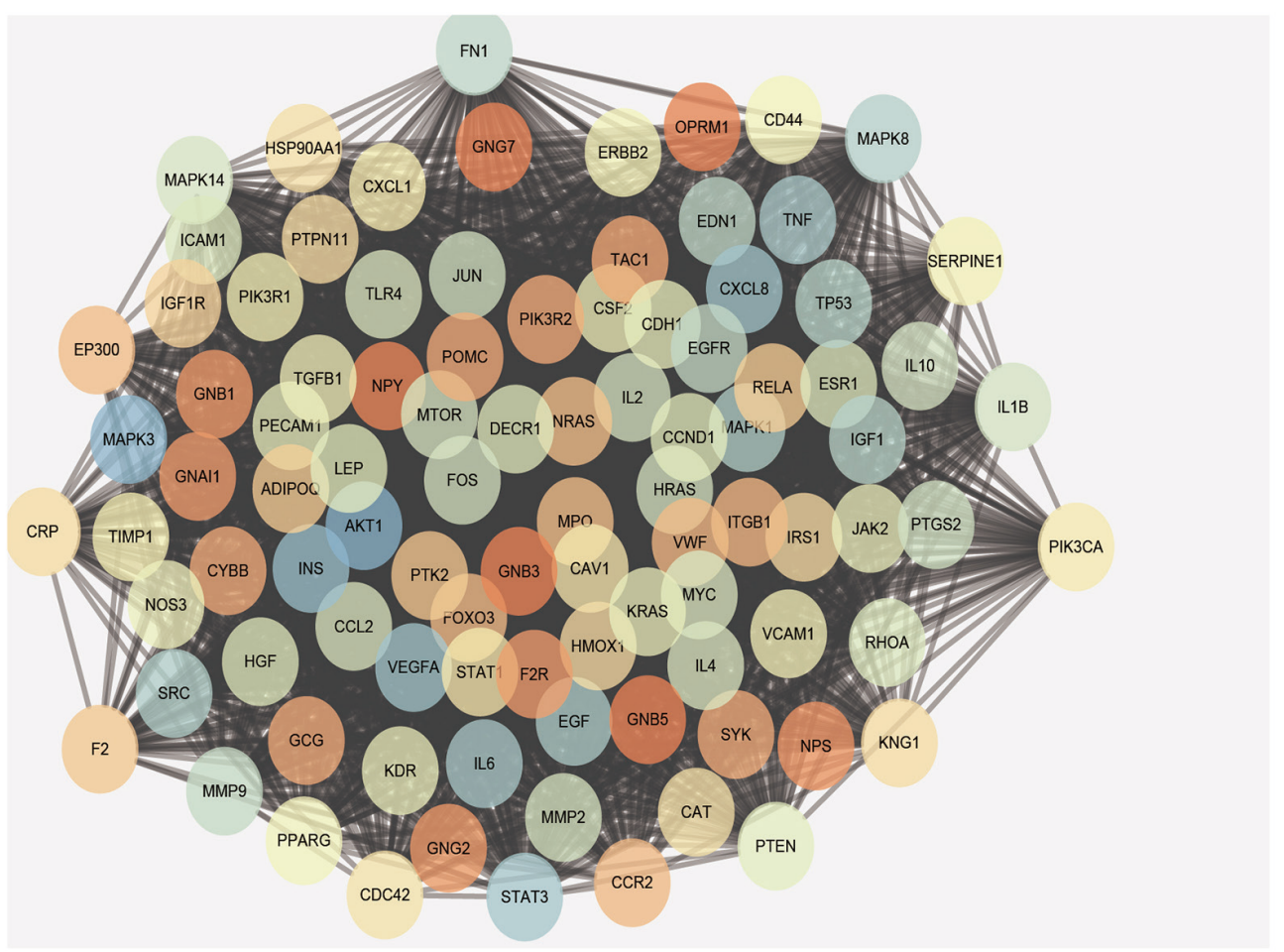

B
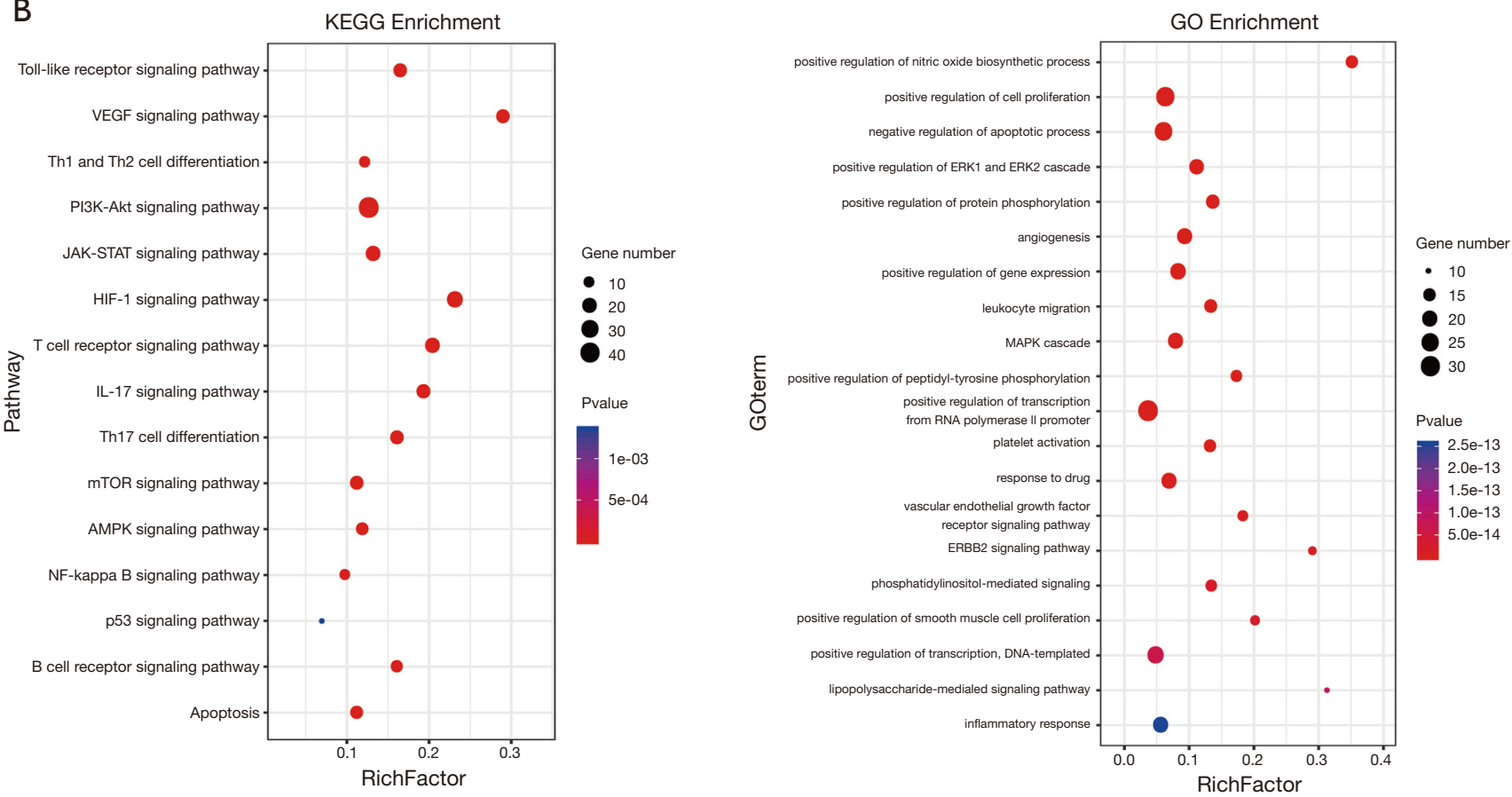

Figure 4 Results ofPPI network of target protein and KEGG enrichment and GO enrichment analysis: (A) Construction of a proteinprotein interaction network expressed by hub the target protein. The node represents the degree: the higher the degree value, the darker the color. (B) KEGG enrichment analysis and GO enrichment analysis. KEGG, Kyoto Encyclopedia of Genes and Genomes; GO, Gene Ontology. 
Table 2 Information on hub targets

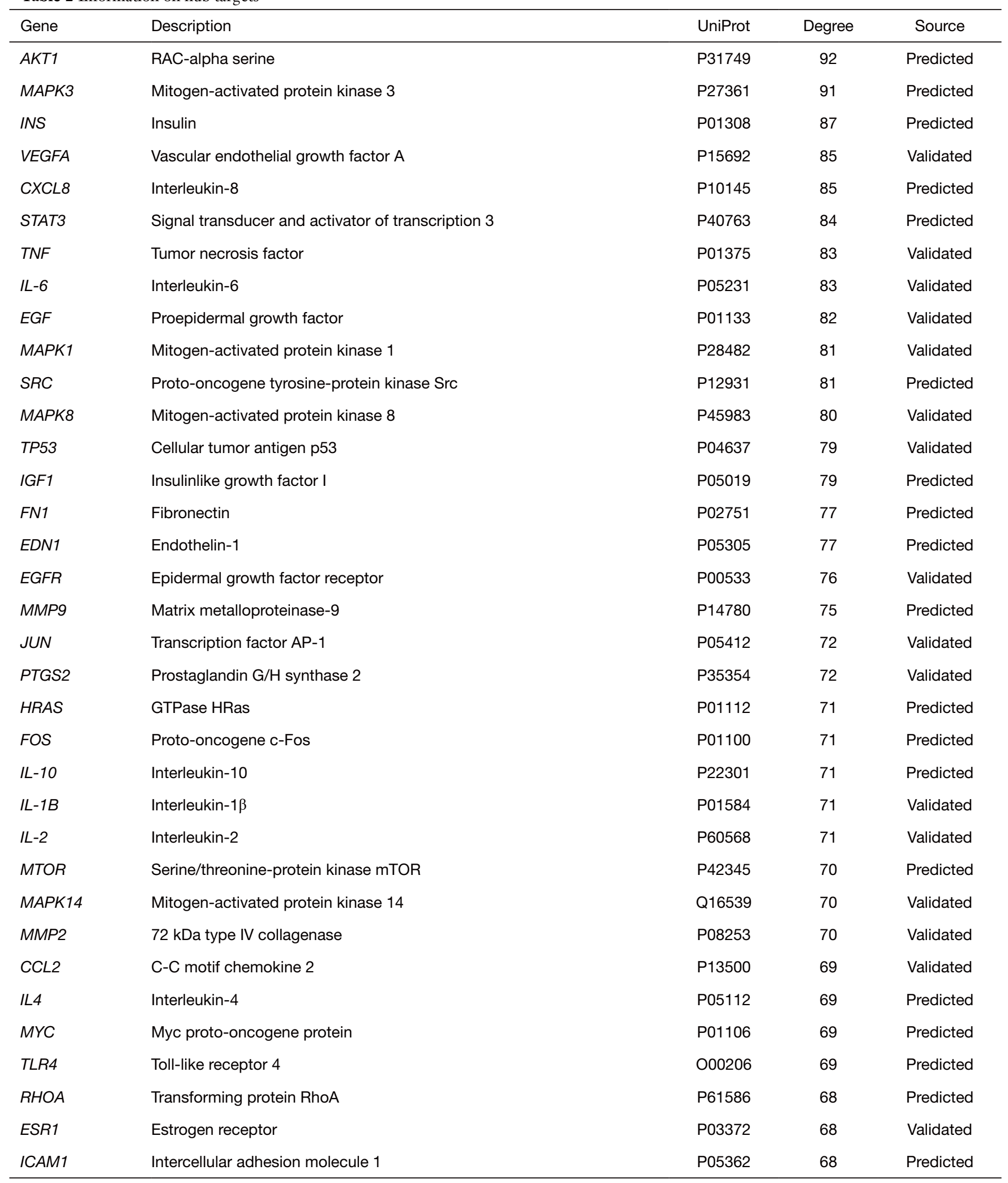

Table 2 (continued) 
Table 2 (continued)

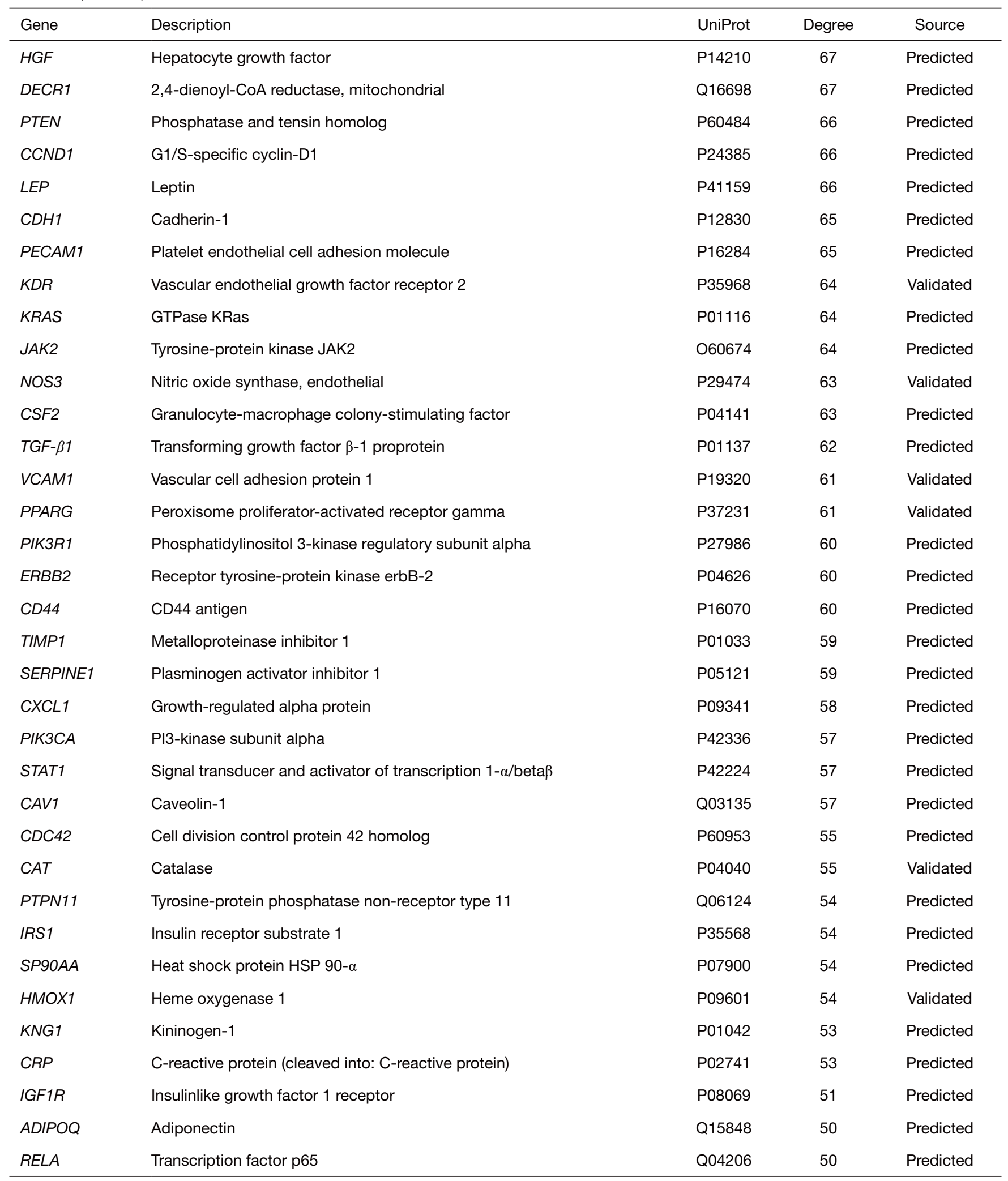

Table 2 (continued) 
Table 2 (continued)

\begin{tabular}{|c|c|c|c|c|}
\hline Gene & Description & UniProt & Degree & Source \\
\hline$F 2$ & Prothrombin & P00734 & 48 & Validated \\
\hline NRAS & GTPase NRas & P01111 & 47 & Predicted \\
\hline MPO & Myeloperoxidase & P05164 & 47 & Validated \\
\hline EP300 & Histone acetyltransferase p300 & Q09472 & 45 & Predicted \\
\hline ITGB1 & Integrin $\beta-1$ & P05556 & 45 & Predicted \\
\hline$V W F$ & von Willebrand factor & P04275 & 45 & Predicted \\
\hline CCR2 & C-C chemokine receptor type 2 & P41597 & 44 & Predicted \\
\hline GCG & Glucagon & P01275 & 41 & Predicted \\
\hline CYBB & Cytochrome b-245 heavy chain & P04839 & 41 & Predicted \\
\hline$S Y K$ & Tyrosine-protein kinase SYK & P43405 & 41 & Predicted \\
\hline TAC1 & Protachykinin-1 & P20366 & 40 & Predicted \\
\hline GNAl1 & Guanine nucleotide-binding protein & P63096 & 37 & Predicted \\
\hline$F 2 R$ & Proteinase-activated receptor 1 & P25116 & 37 & Predicted \\
\hline GNB1 & Guanine nucleotide-binding protein & P62873 & 36 & Predicted \\
\hline GNG2 & Guanine nucleotide-binding protein & P59768 & 36 & Predicted \\
\hline OPRM1 & Mu-type opioid receptor & P35372 & 30 & Validated \\
\hline
\end{tabular}

regulating inflammation and immune response in vivo, Chinese herbal medicine functions through a variety of natural compounds acting on multiple targets; thus, the mechanism of action is particularly complex and difficult to explain. Therefore, it is of considerable value using network pharmacology based on big data bio-informatics to gain deeper understanding of the molecular mechanism of Chinese herbal medicine in treating certain diseases.

According to our herbal-compound-target network diagram, 4 core compounds, quercetin, kaempferol, betasitosterol, and stigmasterol, were identified. Quercetin is a plant-derived flavonoid (chemical name: 3,3',4',5,7-pe ntahydroxyflavonoid) which has a good antioxidative and anti-inflammatory effects. Most plant-derived secondary metabolites can directly affect the expression of key proinflammatory molecules in inflammatory mediators (17). Meanwhile, kaempferol is a natural flavonoid compound isolated from plants. Kaempferol can effectively inhibit the further development of allergic inflammation of the intestinal cell model mediated by immunoglobulin $\mathrm{E}(\mathrm{IgE})$ in vivo by inhibiting the secretion of allergic media. Flavonol fisetin can improve the AHR of asthma, which is related 

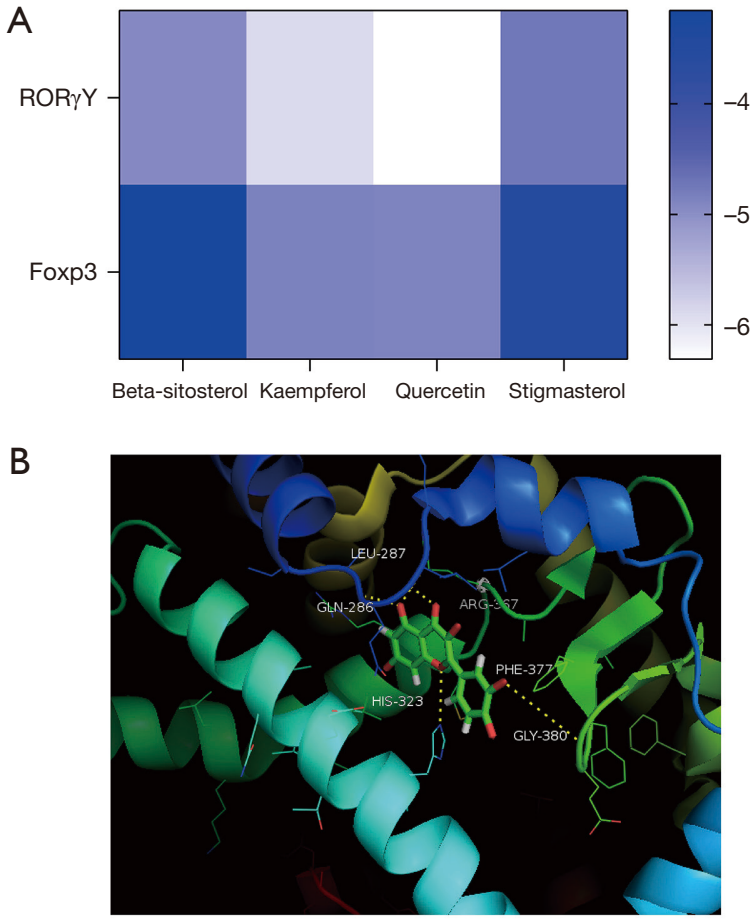

Figure 5 Results of Molecular docking. (A) The thermogram shows a large amount of statistical data on the binding of Foxp3 and RoR $\gamma$ t to the core compounds of PFQF. (B) Schematic diagram of quercetin and RoR $\gamma$ t docking. PFQF, Ping Feng Qingfei Mixture.

to reducing the Th2 type response and inhibiting necrosis factor (NF) $-\kappa \mathrm{B}$ and its downstream chemokines (18). Beta-sitosterol can increase Th1 cell activity and inhibit Th2 cell activity, and thus has a clear inhibitory effect on airway inflammation induced by allergens (19). Finally, stigmasterol is a natural sterol belonging to a large group of phytosterols. Studies have shown that it has the ability to inhibit airway inflammation and remodeling by inhibiting the response mediated by $\operatorname{IgE}$ induced by allergens (20). These compounds are the material basis of PFQF in the treatment of AHR.

According to the analysis of KEGG and GO enrichment, we speculated that PFQF's treatment effect involves the regulation of Th17-Treg imbalance, and subsequent molecular docking verified confirmed this assumption. In recent years, Th17-Treg immune imbalance has been found to be an important mechanism, along with that of Th1/Th2 (21). Both Th17 and Treg immune cells are derived from cluster of differentiation (CD)4+ $\mathrm{T}$ cells, which are closely related to the differentiation process.
Th17-Treg imbalance may play a key role in allergic airway inflammation (22). The increase of Th17 level promotes the inflammatory response, while the decrease of Treg level leads to a decrease of the ability of the body to inhibit an inflammatory response (23). Th17 immune cells are T lymphocyte subsets which are different from both Th1 and Th2 immune cells, and could also cause AHR (24). The specific marker of Th17 immune cells is ROR $\gamma \mathrm{T}$, which can produce many inflammatory factors, including IL-17 and IL-6, that in turn can enhance the eosinophilic airway inflammation mediated by Th2 cells (25). Studies have shown that IL-17A, an inflammatory factor in Th17 cells, can stimulate airway epithelial cells, stromal cells, and endothelial cells to secrete related proinflammatory factors, leading to the activation of neutrophils, and ultimately leading to asthma inflammation (26). These cells can secrete IL-4, IL-10, and transforming growth factor $\beta-1$ (TGF- $\beta$ ). All these cytokines have anti-inflammatory effects, which can inhibit the hyperactive Th2-type reaction. The FOXP3 gene is a specific transcription regulator of Treg, which can promote the differentiation of immature CD4 cells into Treg cell subsets. If the FOXP3 gene is mutated, it can affect the development and maturation of Treg cells (27). It has been reported that infusion of nonantigen-sensitized Treg cells into mice can reduce AHR in asthmatic mice (28), and other studies have shown that the Th17:Treg ratio is positively correlated with AHR (25).

A major feature of Th17 cells is the production of inflammatory factor IL-17. It is an activator of neutrophils and is involved in the process of airway inflammation and remodeling (29). IL-6 plays a key role in inflammation and immunity. The expression level of IL-6 is closely related to AHR, and the secretion of IL-6 figures prominently in the pathogenesis of AHR (30). Furthermore, IL-6 can induce immature Th0 cells to polarize Th17, especially to RoR- $\gamma$ T by activating the STAT3 target gene (31). L-10 cytokines are produced by Treg immune cells, which have antiinflammatory effects, and L-10 is an important cytokine for the ability of Tregs to inhibit AHR (32). This includes not only inhibiting the infiltration of eosinophils in the airway but also achieving immune tolerance and protecting the body's airway during asthma (33). In addition, IL-10 levels in asthmatic patients are generally low (34). It has been reported that TGF- $\beta$ is positively correlated with FOXP3 expression (35). TGF- $\beta$ can regulate the differentiation of Treg and Th17 cells and inhibit the proliferation and expression of most immune cells (36). 


\section{Conclusions}

We systematically explored the pharmacological mechanism of PFQF in the treatment of AHR caused by PM2.5 through network pharmacology and molecular docking. Network pharmacology is uniquely suited to clarify the therapeutic mechanism of TCM. Through network pharmacology, we discovered that PFQF can mitigate AHR through the Th17 cell differentiation and IL-17 signaling pathway. Later molecular docking also confirmed the regulation of Th17-Treg imbalance by PFQF. We hope our study can act as an exemplar for modern research into $\mathrm{TCM}$ and that PFQF can provide an alternative treatment for haze-related diseases.

\section{Acknowledgments}

Funding: This research was funded by the key R \& D program of Shandong Province (No. 2016GSF202038).

\section{Footnote}

Conflicts of Interest: All authors have completed the ICMJE uniform disclosure form (available at http://dx.doi. org/10.21037/apm-21-802). The authors have no conflicts of interest to declare.

Ethical Statement: The authors are accountable for all aspects of the work in ensuring that questions related to the accuracy or integrity of any part of the work are appropriately investigated and resolved. The study was conducted in accordance with the Declaration of Helsinki (as revised in 2013).

Open Access Statement: This is an Open Access article distributed in accordance with the Creative Commons Attribution-NonCommercial-NoDerivs 4.0 International License (CC BY-NC-ND 4.0), which permits the noncommercial replication and distribution of the article with the strict proviso that no changes or edits are made and the original work is properly cited (including links to both the formal publication through the relevant DOI and the license). See: https://creativecommons.org/licenses/by-nc-nd/4.0/.

\section{References}

1. Borak J, Lefkowitz RY. Bronchial hyperresponsiveness. Occup Med (Lond) 2016;66:95-105.
2. O'Byrne PM, Inman MD. Airway hyperresponsiveness. Chest 2003;123:411S-6S.

3. Meurs H, Gosens R, Zaagsma J. Airway hyperresponsiveness in asthma: Lessons from in vitro model systems and animal models. Eur Respir J 2008;32:487-502.

4. Gon Y, Hashimoto S. Role of airway epithelial barrier dysfunction in pathogenesis of asthma. Allergol Int 2018;67:12-7.

5. Nasab EM, Athari SM, Ghafarzade S, et al. Immunomodulatory effects of two silymarin isomers in a Balb/c mouse model of allergic asthma [published online ahead of print, 2020, Apr 10]. AllergolImmunopathol (Madr) 2020;S0301-054630029-X.

6. Xu M, Zhang Y, Wang M, et al. TRPV1 and TRPA1 in lung inflammation and airway hyperresponsiveness induced by fine particulate matter (PM2.5). Oxid Med Cell Longev 2019;2019:7450151.

7. Xu M, Li F, Wang M, et al. Protective effects of VGX1027 in PM2.5-induced airway inflammation and bronchial hyperresponsiveness. Eur J Pharmacol 2019;842:373-83.

8. Schlesinger RB. The health impact of common inorganic components of fine particulate matter (PM2.5) in ambient air: A critical review. Inhal Toxicol 2007;19:811-32.

9. $\mathrm{Li} \mathrm{K}, \mathrm{Li} \mathrm{H}, \mathrm{Xu} \mathrm{W}$, et al. Research on the potential mechanism of gypenosides on treating thyroid-associated ophthalmopathy based on network pharmacology. Med Sci Monit 2019;25:4923-32.

10. Song W, Ni S, Fu Y, et al. Uncovering the mechanism of maxing Ganshi decoction on asthma from a systematic perspective: A network pharmacology study. Sci Rep 2018;8:17362.

11. Wu T, Yue R, Li L, et al. Study on the mechanisms of Banxia Xiexin decoction in treating diabetic gastroparesis based on network pharmacology. Interdiscip Sci 2020;12:487-98.

12. Lu Y, Sun J, Hu M, et al. Network pharmacology analysis to uncover the potential mechanisms of lyciumbarbarum on colorectal cancer. Interdiscip Sci 2020;12:515-25.

13. Hu W, Fu W, Wei X, et al. A network pharmacology study on the active ingredients and potential targets of tripterygium wilfordii hook for treatment of rheumatoid arthritis. Evid Based Complement Alternat Med 2019;2019:5276865.

14. Wang W, Liu T, Yang L, et al. Study on the multi-targets mechanism of triphala on cardio-cerebral vascular diseases based on network pharmacology. Biomed Pharmacother 2019;116:108994.

15. Xuan X, Sun Z, Yu C, et al. Network pharmacology-based 
study of the protective mechanism of conciliatory antiallergic decoction on asthma. Allergol Immunopathol (Madr) 2020;48:441-9.

16. Lin Y, Zou J, Yang W, et al. A review of recent advances in research on PM2.5 in China. Int J Environ Res Public Health 2018;15:438.

17. Rogerio AP, Kanashiro A, Fontanari C, et al. Antiinflammatory activity of quercetin and isoquercitrin in experimental murine allergic asthma. Inflamm Res 2007;56:402-8.

18. Gong JH, Shin D, Han SY, et al. Blockade of airway inflammation by Kaempferol via disturbing Tyk-STAT signaling in airway epithelial cells and in asthmatic mice. Evid Based Complement Alternat Med 2013;2013:250725.

19. Mahajan SG, Mehta AA. Suppression of ovalbumininduced Th2-driven airway inflammation by $\beta$-sitosterol in a guinea pig model of asthma. Eur J Pharmacol 2011;650:458-64.

20. Antwi AO, Obiri DD, Osafo N. Stigmasterol modulates allergic airway inflammation in guinea pig model of ovalbumin-induced asthma. Mediators Inflamm 2017;2017:2953930.

21. Li Y, Li H, Cao Y, et al. Placenta-derived mesenchymal stem cells improve airway hyperresponsiveness and inflammation in asthmatic rats by modulating the Th17/ Treg balance. Mol Med Rep 2017;16:8137-45.

22. Zhao J, Lloyd CM, Noble A. Th17 responses in chronic allergic airway inflammation abrogate regulatory T-cellmediated tolerance and contribute to airway remodeling. Mucosal Immunol 2013;6:335-46.

23. Noval Rivas M, Chatila TA. Regulatory T cells in allergic diseases. J Allergy Clin Immunol 2016;138:639-52.

24. Lin CC, Wang YY, Chen SM, et al. SheganMahuang decoction ameliorates asthmatic airway hyperresponsiveness by downregulating Th2/Th17 cells but upregulating CD4+FoxP3+ Tregs. J Ethnopharmacol 2020;253:112656.

25. Zheng R, Wang F, Huang Y, et al. Elevated Th17 cell frequencies and Th17/Treg ratio are associated with airway hyperresponsiveness in asthmatic children. J Asthma 2020;13:1-10.

26. Movassagh H, Shan L, Mohammed A, et al. Semaphorin $3 \mathrm{E}$ deficiency exacerbates airway inflammation, hyperresponsiveness, and remodeling in a mouse model of allergic asthma. J Immunol 2017;198:1805-14.

27. Dang EV, Barbi J, Yang HY, et al. Control of T(H)17/ $\mathrm{T}$ (reg) balance by hypoxia-inducible factor 1 . Cell 2011;146:772-84.
28. Joetham A, Takeda K, Taube C, et al. Naturally occurring lung CD4(+)CD25(+) T cell regulation of airway allergic responses depends on IL-10 induction of TGF-beta (published correction appears in J Immunol 2007 Apr 15;178(8):5400. Takada, Katsuyuki [corrected to Takeda, Katsuyuki]; Matsubara, Satoko [corrected to Matsubara, Shigeki]). J Immunol 2007;178:1433-42.

29. Wang J, Kong L, Luo Q, et al. Dual effects of respiratory syncytial virus infections on airway inflammation by regulation of Th17/Treg responses in ovalbuminchallenged mice. Inflammation 2014;37:1984-2005.

30. Kuhn C 3rd, Homer RJ, Zhu Z, et al. Airway hyperresponsiveness and airway obstruction in transgenic mice. Morphologic correlates in mice overexpressing interleukin (IL)-11 and IL-6 in the lung. Am J Respir Cell Mol Biol 2000;22:289-95.

31. Yamashita T, Iwakura T, Matsui K, et al. IL-6-mediated Th17 differentiation through ROR $\gamma \mathrm{t}$ is essential for the initiation of experimental autoimmune myocarditis. Cardiovasc Res 2011;91:640-8.

32. Yang N, Shang YX. Epigallocatechin gallate ameliorates airway inflammation by regulating Treg/Th17 imbalance in an asthmatic mouse model. Int Immunopharmacol 2019; $72: 422-8$.

33. Koya T, Matsuda H, Takeda K, et al. IL-10-treated dendritic cells decrease airway hyperresponsiveness and airway inflammation in mice. J Allergy Clin Immunol 2007;119:1241-50.

34. Raeiszadeh Jahromi S, Mahesh PA, Jayaraj BS, et al. Serum levels of IL-10, IL-17F and IL-33 in patients with asthma: A case-control study. J Asthma 2014;51:1004-13.

35. Mantel PY, Kuipers H, Boyman O, et al. GATA3-driven Th2 responses inhibit TGF-beta1-induced FOXP3 expression and the formation of regulatory T cells. PLoS Biol 2007;5:e329.

36. Gordon ED, Sidhu SS, Wang ZE, et al. A protective role for periostin and TGF- $\beta$ in IgE-mediated allergy and airway hyperresponsiveness. Clin Exp Allergy 2012;42:144-55.

(English Language Editor: J. Gray)

Cite this article as: Wang $M$, Peng $M$, Shi H, Dong C, Cui L, Chang H, Kan Z, Zhen K, Si G, Li H. Ping Feng Qingfei mixture treats airway hyperresponsiveness: a network pharmacology and molecular docking study. Ann Palliat Med 2021;10(4):4747-4759. doi: 10.21037/apm-21-802 